\title{
miR-222 regulates the cell biological behavior of oral squamous cell carcinoma by targeting PUMA
}

\author{
FANGFANG JIANG* ${ }^{*}$ WEI ZHAO* , LIJIE ZHOU, LIN ZHANG, ZIFENG LIU and DONGSHENG YU \\ Institute of Stomatological Research, Department of Oral and Maxillofacial Surgery, Guanghua College of Stomatology, \\ Sun Yat-sen University, Guangzhou, Guangdong 510055, P.R. China
}

Received November 15, 2013; Accepted December 27, 2013

DOI: $10.3892 /$ or.2014.2985

\begin{abstract}
Previous reports have shown that low expression of p53 upregulated modulator of apoptosis (PUMA) and abnormal expression patterns of a number of miRNAs may be associated with poor prognosis in various types of human malignancies. As a member of the oncomiRs, miR-222 has been found to be upregulated in oral squamous cell carcinoma (OSCC). We hypothesized that there was an important relationship between miR-222 and PUMA in OSCC based on the prediction of the target genes of miR-222. In the present study, Pre-miR-222, As-miR-222 and the empty vector, were used to treat OSCC cells, respectively. Using the non-transfected cells as blank control, the expression levels of miR-222 and the PUMA gene were evaluated by RT-PCR and western blotting. Cell proliferation and migration abilities were analyzed by MTT and Transwell assays. Cell cycle distribution and apoptosis were assessed by flow cytometry. Our results demonstrated that, when compared with the blank control group, OSCC cells in the Pre-miR-222 transfection group showed increased expression of miR-222 and decreased expression of PUMA, enhanced proliferation and invasion abilities, and decreased apoptosis. In contrast, the above indices in the As-miR-222 transfection group confirmed the opposite results when compared with those in the Pre-miR-222 transfection group. In addition, no significant differences between the empty vector transfection group and the control group were noted. Our results suggest that miR-222 targets the expression of PUMA in OSCC cells and affects cell growth, invasive and apoptotic abilities. Thus, PUMA may be a possible new target for the treatment of OSCC.
\end{abstract}

Correspondence to: Professor Dongsheng Yu, Institute of Stomatological Research, Department of Oral and Maxillofacial Surgery, Guanghua College of Stomatology, Sun Yat-sen University, 56 Lingyuan Road West, Guangzhou, Guangdong 510055, P.R. China E-mail: yudsh@mail.sysu.edu.cn

*Contributed equally

Key words: oral squamous cell carcinoma, miR-222, PUMA, proliferation, apoptosis

\section{Introduction}

MicroRNAs (miRNAs), which play important roles in transformation and carcinogenesis, are potent post-transcriptional regulators of protein-coding genes and function as tumor oncogenes or suppressors $(1,2)$. As a member of the oncomiRs, miR-222 has been reported to drive the oncogenesis of many types of malignancies (3-5), and a recent study demonstrated that co-suppression of miR-221/222 inhibits glioma cell growth by targeting the $3^{\prime}$-untranslated region (3'UTR) of p27 mRNA in vitro and in vivo (4). However, the mode of action of miR-222 in carcinogenesis has not been characterized, and the mechanism of the biological function of miR-222 in oral squamous cell carcinoma (OSCC) remains unknown.

The p53 upregulated modulator of apoptosis (PUMA), also called Bcl-2 binding component 3 (BBC3), was newly discovered as a target for activation by p53 in 2001 and was found to possess a powerful pro-apoptotic effect as a member of the Bcl-2 family (6-8). Although the specific mechanisms for inducing apoptosis require further investigation, PUMA is a promising new target for gene therapy as its pro-apoptotic function has achieved favorable results $(1,2,9)$.

Studies suggest that PUMA is a direct target of miR-222 which functions as an endogenous apoptosis regulator in various common forms of human epithelial cancers $(4,5)$. However, little is known concerning the role of PUMA in the targeted treatment of human OSCC. In the present study, we identified miR-222 as a potent regulator of PUMA, a cell proliferation inducer and tumor promoter. Inhibition of miR-222 appears to induce cell apoptosis and reduce cell growth and directly upregulate PUMA expression by targeting the binding sites in the $3^{\prime}$ UTR. The results suggest that miR-222 may play important roles in regulating the cell biological behavior of OSCC by targeting PUMA.

\section{Materials and methods}

Cell culture and transfection. The OSCC cell lines Tca8113 and UM1 were cultured in Dulbecco's modified Eagle's medium (DMEM) supplemented with 10\% fetal bovine serum (FBS), penicillin and streptomycin $(100 \mu \mathrm{g} / \mathrm{ml})$, and incubated in a humidified $5 \% \mathrm{CO}_{2}$ environment at $37^{\circ} \mathrm{C}$. The 2'-OMe-oligonucleotides were synthesized and purified by high-performance liquid chromatography by GenePharma 
Co. Ltd. (Shanghai, China). The oligonucleotides were modified by 2'-OMe to obtain the following sequences: hsa-miR-222 inhibitor (As-miR-222), 5'-ACCCAGUAGCCAGAUGUAG CU-3'; hsa-miR-222 mimics (Pre-miR-222), the positive-sense strand, 5'-AGCUACAUCUGGCUACUGGGU-3', and the antisense strand, 5'-CCAGUAGCCAGAUGUAGCUUU-3'; and the empty vector, 5'-CAGUACUUUUGUGUAGUACAA-3'. Pre-miR-222, As-miR-222 and the empty vector were transfected using Lipofectamine ${ }^{\mathrm{TM}}$ RNAiMAX (Invitrogen) according to the recommended protocol, and the transfection medium was replaced with fresh medium 4-6 h later. After treatment for 2 days, cells were divided into 4 groups: the control, empty vector, Pre-miR-222 and As-miR-222 which were used for subsequent analysis.

RNA preparation and reverse transcription-polymerase chain reaction ( $R T-P C R)$. After treatment for $48 \mathrm{~h}$, Tca8113 and UM1 cells were lysed using TRIzol reagent (Invitrogen), and total RNA was extracted using an RNeasy Mini kit according to the manufacturer's instructions. RT-PCR was carried out using a reverse transcription kit, and the human $\beta$-actin gene was used as the internal control. The PUMA gene primers were as follows: 5'-TGTCGAATAAACGCTTTACAAAC-3' (forward) and 5'-AACGTTTGTAATGATGGCTTCTG-3' (reverse). The $\beta$-actin primers were as follows: 5'-GGT CGGAGTCAACGGATTTGGTCG-3' (forward) and 5'-CCT CCGACGCCTGCTTCACCAC-3' (reverse). Relative expression levels were calculated using the $2^{-\Delta \Delta C t}$ method (10).

Western blot analysis. After transfection for $48 \mathrm{~h}$, cells of every group were washed with cold phosphate-buffered saline (PBS) three times and lysed in lysis buffer. Cell lysates were centrifuged at $14,000 \mathrm{x}$ g for $20 \mathrm{~min}$ at $4^{\circ} \mathrm{C}$, and the protein concentration was determined using the Enhanced BCA protein assay kit (Beyotime Institute of Biotechnology, China) using bovine serum albumin (BSA) as the standard. Equal amounts of crude protein were mixed with sodium dodecyl sulphate (SDS) sample buffer and denatured and then separated on $12 \%$ SDS-polyacrylamide gels. After electrophoresis, gels were transferred to a PVDF membrane by electroblotting. The membrane was blocked in Tris-buffered saline (TBST) (50 mM Tris- $\mathrm{HCl} \mathrm{pH} 7.5,150 \mathrm{mM} \mathrm{NaCl}, 0.2 \%$ Tween) containing 5\% skim milk for $1 \mathrm{~h}$ at room temperature. The blots were incubated with the primary antibodies against PUMA, Bcl-2 and GAPDH overnight at $4^{\circ} \mathrm{C}$ and rinsed three times with TBST. The rinsed blots were then incubated with the secondary antibody for $1 \mathrm{~h}$ and washed with TBST. The membrane was developed by enhanced chemiluminescence and exposed to AlphaView SA for 1-15 min. The bands of the specific proteins were quantified after normalization with the density of GAPDH with ImageJ instrument software.

Immunofluorescence. Tca8113 and UM1 cells were seeded in 24-well plates with $12-\mathrm{mm}$-diameter coverslips. After incubation for at least $24 \mathrm{~h}$, the cells were treated with Pre-miR-222, As-miR-222 or empty vector for the indicated times. Subsequently, cells were washed three times with PBS ( $\mathrm{pH} 7.4$ ), fixed in $4 \%$ formaldehyde for $20 \mathrm{~min}$ and permeabilized in $0.1 \%$ Triton X-100 for $30 \mathrm{~min}$ and sequentially washed three times with PBS. The cells were then incubated with the indicated primary antibodies (PUMA and Bcl-2) according to the manufacturer's instructions, and the primary antibodies were diluted $(1: 250)$ in $1 \mathrm{X}$ PBS as recommended by the manufacturer. After $18 \mathrm{~h}$, cells were washed three times with PBS and incubated for $1 \mathrm{~h}$ with each of the corresponding secondary antibodies. Nuclei were stained with the nuclear stain DAPI. After mounting with the coverslips on the microscopic slides, cells were examined using laser scanning confocal microscopy.

Cell migration assay. After transfection, OSCC cells in $\mathrm{DMEM} / \mathrm{F} 12$ medium containing $0.1 \%$ FBS were transferred to $8-\mu \mathrm{m}$ pore inserts, and then placed in companion wells which contained DMEM/F12 medium and 10\% FBS as a chemoattractant. The inserts were removed after a 12-h incubation, and the non-migrating OSCC cells on the upper surface were harvested. Cells on the lower surface were fixed and stained and then counted under a microscope.

Cell viability assay. The OSCC Tca8113 and UM1 cell lines were seeded into 96-well plates with $\sim 4,000$ cells/well. After transfection as described above, $10 \mu \mathrm{l}$ of 3-(4,5-dimethylthiazol-2-yl)-2,5-diphenyltetrazolium bromide (MTT) $(5 \mathrm{mg} / \mathrm{ml})$ was added to each well at 12, 24, 36, 48, 60 and $72 \mathrm{~h}$ after treatment and incubated for $4 \mathrm{~h}$ at $37^{\circ} \mathrm{C}$. The supernatant was discarded, and $100 \mu \mathrm{l}$ of DMSO was added to each well to dissolve the precipitate. Optical density (OD) was measured at the wavelength of $570 \mathrm{~nm}$. The data are expressed as means $\pm \mathrm{SD}$, derived from quintuplicate samples of at least three independent experiments.

Apoptosis assays. Treated and untreated cells were washed with cold PBS twice and resuspended in buffer at a concentration of $10^{6} / \mathrm{ml}$. Cells were mixed with $5 \mu \mathrm{l}$ of FITC-conjugated Annexin $\mathrm{V}$ reagent and $5 \mu \mathrm{l}$ of propidium iodide (PI). After a 15-min incubation at room temperature in the dark, samples were analyzed by flow cytometry.

Statistical analysis. The experimental data are presented as means \pm SD from at least triplicate analyses. Statistical analyses were performed by one-way analysis of variance (ANOVA) or t-test using SPSS software 17.0. Statistical significance was set at $\mathrm{P}<0.05$.

\section{Results}

Expression of miR-222 and PUMA in OSCC cells. In the transfection groups of Tca8113 and UM1 cells, As-miR-222 inhibited the expression of miR-222. In order to knock down endogenous miR-222, chemically engineered oligonucleotides were synthesized and transfected into Tca8113 and UM1 cells. RT-PCR analysis showed that As-miR-222 efficiently and specifically silenced endogenous miR-222. In contrast, the expression of the PUMA gene in the As-miR-222 group was upregulated to a greater extent when compared to the control group. However, cells transfected with Pre-miR-222 exhibited an opposite trend of expression. No significant differences between the empty vector transfection group and the control group were noted. The results showed that miR-222 was negatively correlated with PUMA expression in 

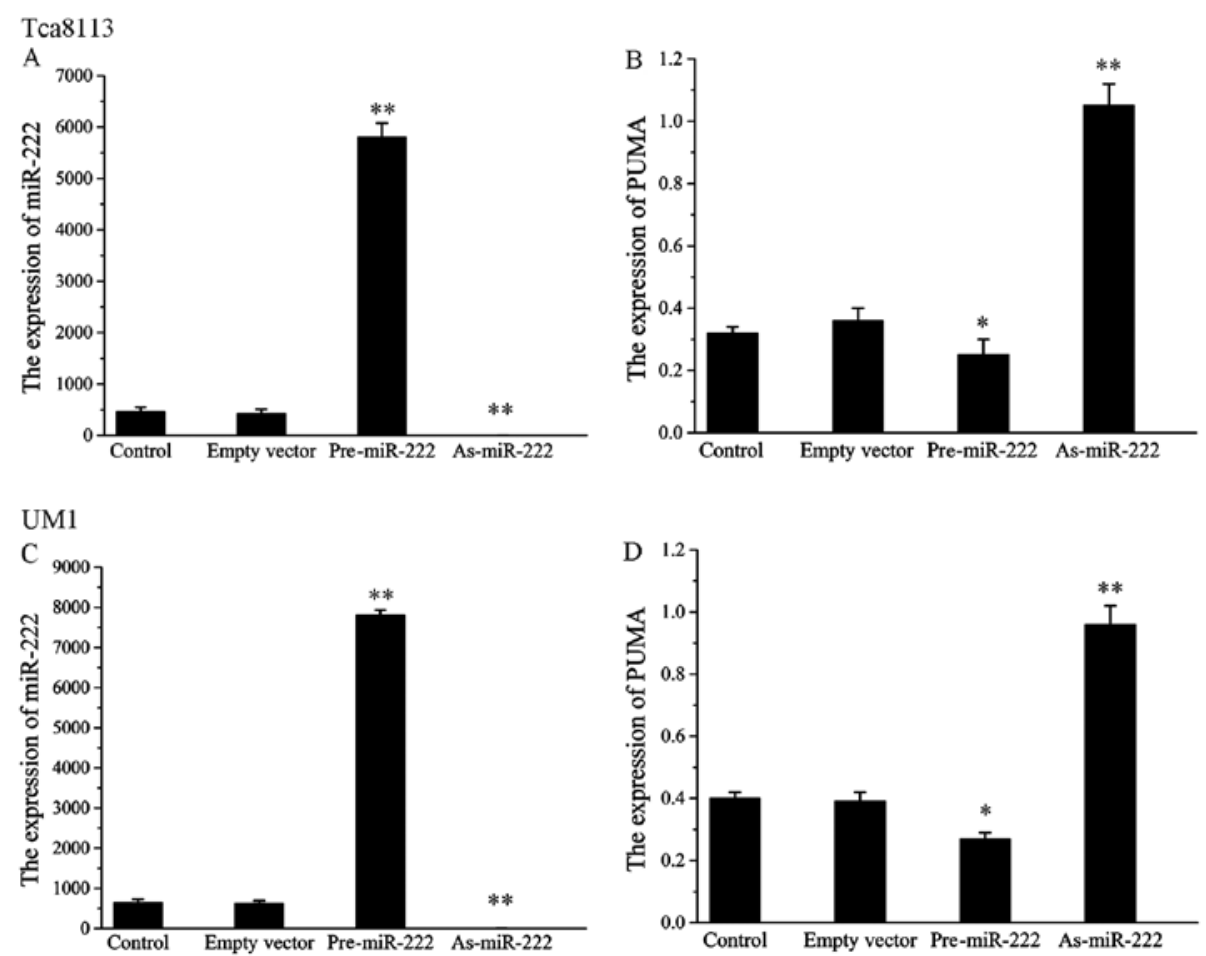

Figure 1. miR-222 regulates the expression of PUMA in Tca8113 and UM1 cells. RT-PCR analysis of the expression of (A and C) miR-222 and (B and D) PUMA in OSCC Tca8113 and UM1 cell lines after treatment with Pre-miR-222, As-miR-222 and empty vector. RT-PCR was performed as described in Materials and methods. PUMA, p53 upregulated modulator of apoptosis; RT-PCR, reverse transcription-polymerase chain reaction; OSCC, oral squamous cell carcinoma.

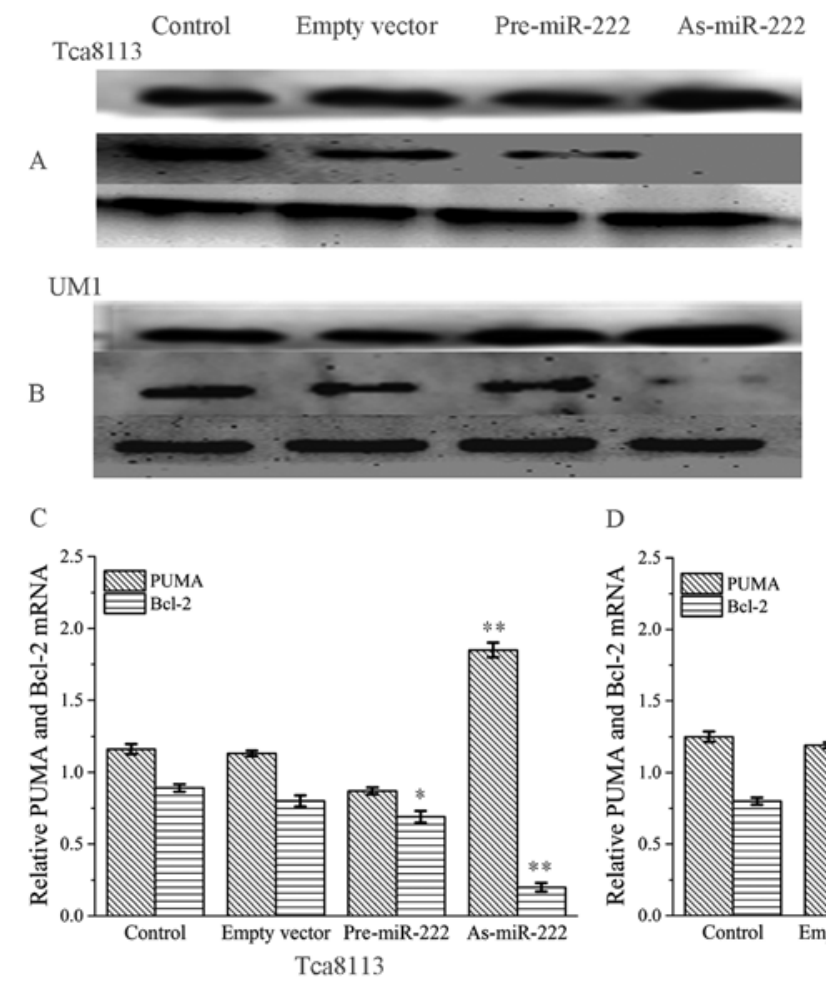

As-miR-222

PUMA

Bcl-2

GAPDH

PUMA

Bcl-2

GAPDH

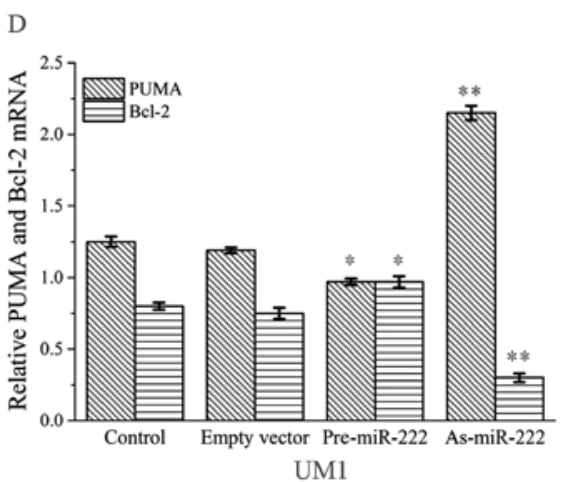

Figure 2. Expression of apoptotic proteins in (A and C) Tca8113 and (B and D) UM1 cells following treatment with Pre-miR-222, As-miR-222 and the empty vector. As determined by western blot assay, PUMA was observed to be overexpressed in the Tca8113 and UM1 cells in the As-miR-222 group, and the expression of PUMA in the Pre-miR-222 group was downregulated to a greater degree when compared with the other three groups. In contrast, the expression of Bcl-2 exhibited an opposite trend when compared with the expression of PUMA. PUMA, p53 upregulated modulator of apoptosis.

the OSCC cell lines. U6 was present as a loading control in the 4 groups (Fig. 1).
Pre-miR-222 and As-miR-222 alter the expression of apoptotic proteins (Fig. 2). The expression of apoptosis-related proteins 

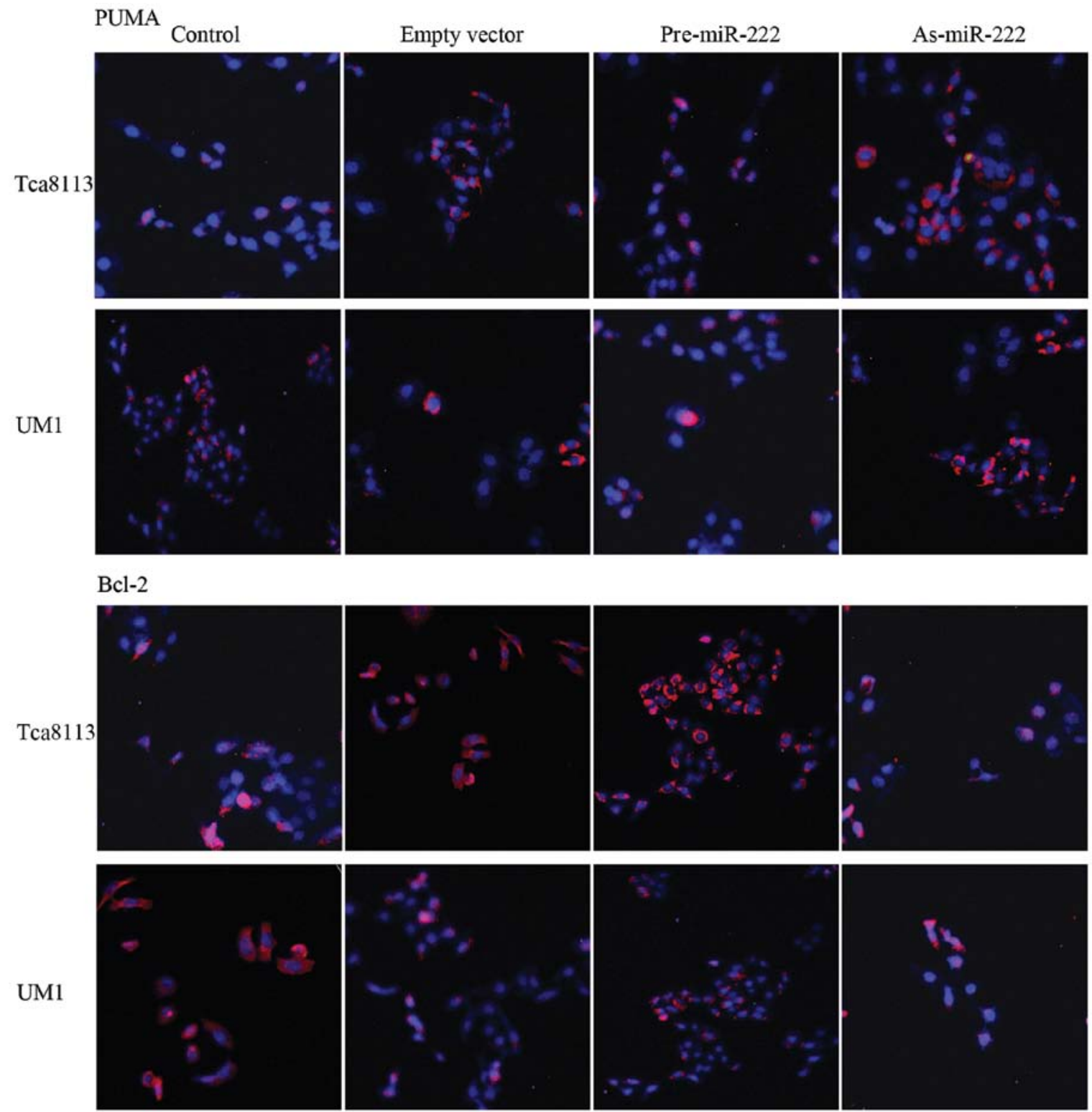

Figure 3. Determination of PUMA and Bcl-2 expression in OSCC cells by immunofluorescence confocal microscopy. Tca8113 and UM1 cells were stained with CY3-conjugated PUMA and Bcl-2 antibodies (red fluorescence), and the nuclei were stained with DAPI (blue fluorescence). Images showed that PUMA was overexpressed in the Tca8113 and UM1 cells following treatment with As-miR-222. However, the expression of PUMA in the Pre-miR-222 group was downregulated to a greater degree when compared to the other three groups. In contrast, Bcl-2 expression in the Pre-miR-222 and As-miR-222 groups exhibited an opposite trend when compared with the expression of PUMA. PUMA, p53 upregulated modulator of apoptosis; OSCC, oral squamous cell carcinoma.

was measured by western blot analysis in order to detect the molecular mechanism of the involvement of miR-222 in the apoptosis of Tca8113 and UM1 cells. A significant increase in PUMA, one of the pro-apoptosis-related proteins, was observed in the Tca8113 and UM1 cells in the As-miR-222 group. In contrast, the expression of Bcl-2 in the As-miR-222 group was significantly downregulated when compared to that in the control group. The data indicate that As-miR-222 induces cancer cell apoptosis through suppression of PUMA and passivation of Bcl-2. Similarly, the Pre-miR-222 group exhibited increased expression of Bcl-2 and decreased expression of PUMA.

Determination of the expression of PUMA and Bcl-2 in Tca8113 and UM1 cells. Since PUMA is one of the pro-apoptosis regulators, overexpression of PUMA has been attributed to the induction of apoptosis. Therefore, in order to determine the expression of PUMA in human OSCC Tca8113 and UM1 cells, we performed immunofluorescence staining and examined the cells under a laser scanning confocal microscope (Fig. 3). Confocal images of OSCC cells after immunofluorescence staining showed high red fluorescence of PUMA (revealed by CY3-conjugate) in the As-miR-222 group, whereas, the Pre-miR-222 group exhibited relatively low red fluorescence suggesting weaker expression of PUMA. In contrast, confocal images showed that the expression of Bcl-2 in the As-miR-222 group was significantly downregulated when compared to that in the control group. However, in the Pre-miR-222 group the expression of $\mathrm{Bcl}-2$ was upregulated. The cell nuclei were stained for blue fluorescence (using DAPI dye). Low expression 

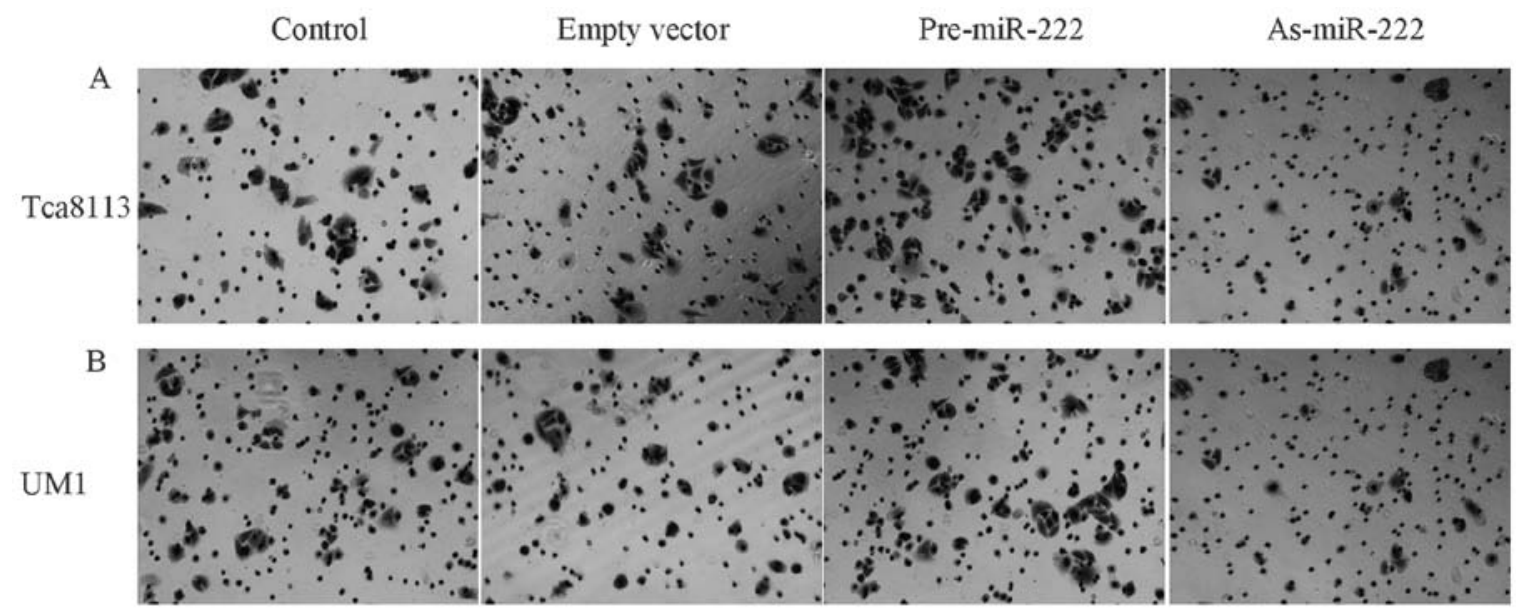

$\mathrm{C}$

$\mathrm{D}$

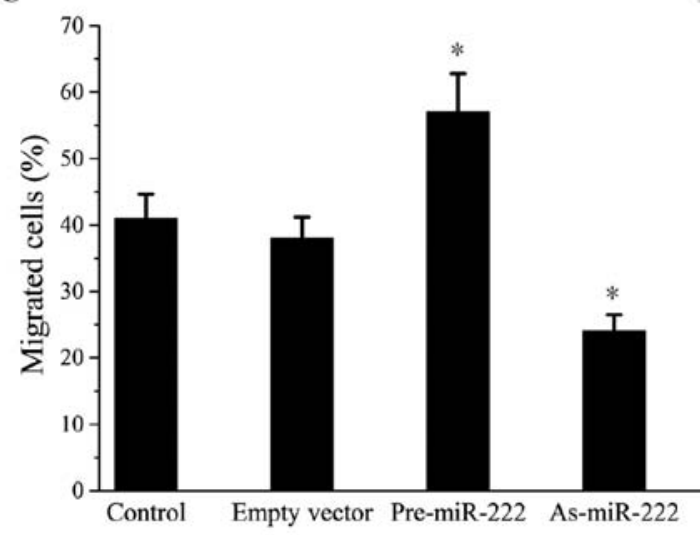

Tca8113

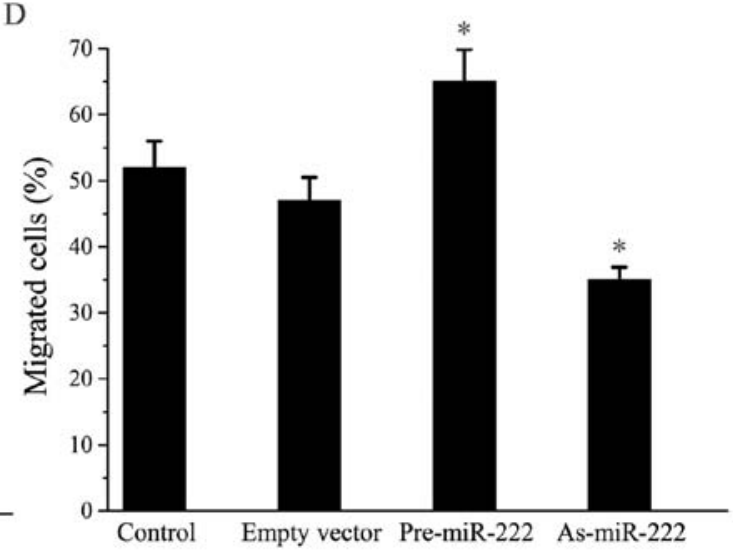

UM1

Figure 4. Effect of miR-222 on the migratory ability of OSCC cells. Images of (A) Tca8113 and (B) UM1 cells which were transferred to inserts following treatment with Pre-miR-222, As-miR-222 and empty vector for migration assays. No significant difference between the empty vector transfection group and the control group in terms of migratory ability was observed. OSCC cells in the Pre-miR-222 transfection group showed enhanced migratory ability. In contrast, the migratory ability of the cells in the As-miR-222 transfection group exhibited an opposite trend when compared with that in the Pre-miR-222 transfection group. (C and D) Data values indicating the percentage of migratory cells are expressed as the means \pm SD of three different experiments. OSCC, oral squamous cell carcinoma.

of PUMA and high expression of Bcl-2 are important characteristics of various types of cancer including OSCC.

Effect of the alteration of miR-222 expression on the migratory ability of OSCC cells. We examined whether an alteration in miR-222 expression is involved in OSCC cell motility, as cell motility plays an important role in metastasis. Cells transfected with Pre-miR-222 or As-miR-222 migrated in a manner which had an obvious difference to that of the control cells (Fig. 4), indicating that alteration of miR-222 did affect the migratory ability of the OSCC cells.

miR-222 influences cancer cell proliferation and apoptosis. As shown in Fig. 5, human OSCC Tca8113 and UM1 cells which were treated with As-miR-222 proliferated at a significantly lower rate than that in the other three groups as evaluated by MTT assay. Apoptosis assays were used to assess whether As-miR-222 inhibits cell proliferation through the induction of cell apoptosis. Annexin V-PI analysis showed that the percentage of apoptotic cells was significantly increased in the cells treated with As-miR-222 as compared to the other three groups, suggesting that apoptosis was obviously induced in cells which were transfected with As-miR-222 (Fig. 6). In the Pre-miR-222 group, both Tca8113 and UM1 cells showed enhanced proliferative ability and decreased apoptosis. Thus, an alteration in miR-222 expression may affect OSCC cell proliferation and apoptosis.

\section{Discussion}

Oral squamous cell carcinoma (OSCC) is one of the most common types of cancer $(11,12)$. Despite improvements in the treatment of OSCC over the last few years, new targeted therapies need to be developed as the survival rate of OSCC patients in the last five years has remained poor $(13,14)$. Therefore, understanding the mechanisms of OSCC and identifying new therapeutic options are clinically significant. As a new strategy, gene therapy was recently developed and has achieved beneficial results.

MicroRNAs (miRNAs) are a class of small non-coding RNAs that negatively regulate gene expression at the post-transcriptional level through association with the 3'-untranslated region (3'UTR) of protein-coding genes and induction of translation inhibition. They play critical roles in the control of cell 

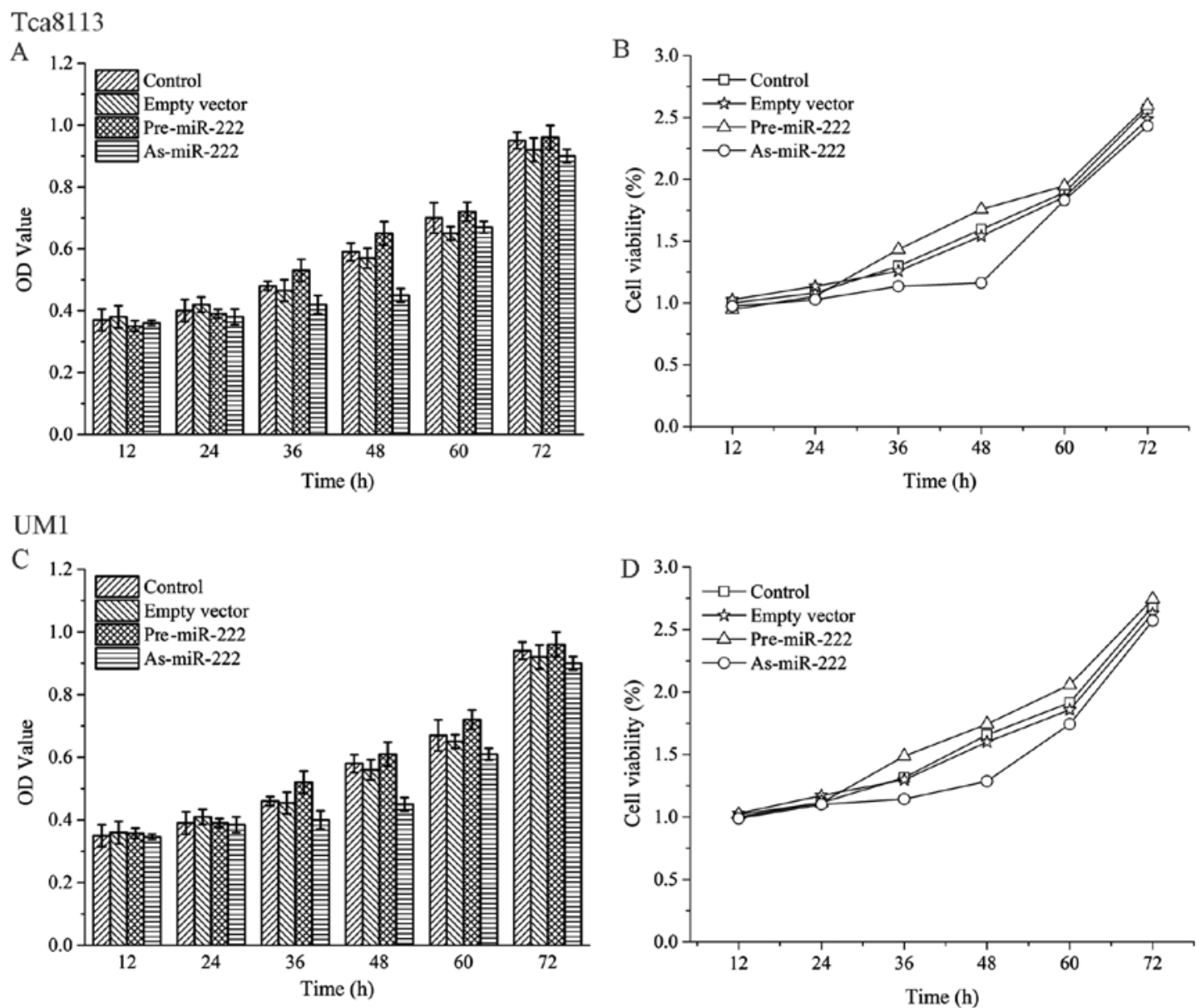

Figure 5. miR-222 influences cancer cell growth. MTT assay showed that (A and B) Tca8113 and (C and D) UM1 cells treated with As-miR-222 proliferated at a significantly lower rate than the other three treatment groups. However, cells in the Pre-miR-222 transfection group exhibited an opposite trend when compared with those in the As-miR-222 transfection group. No significant differences between the empty vector transfection group and the control group were noted.

A

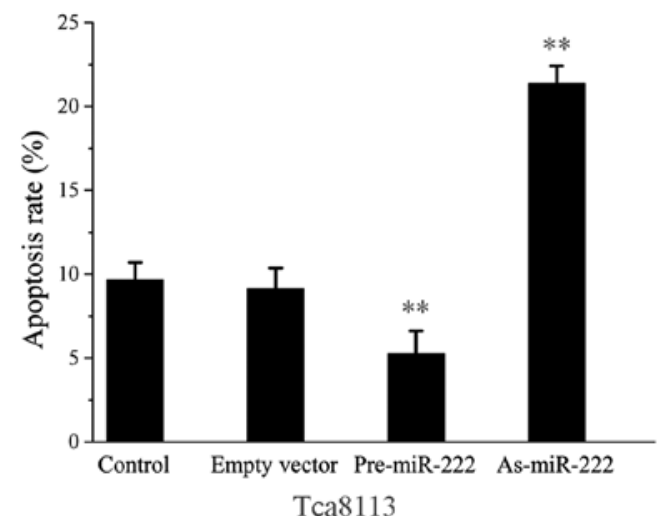

B

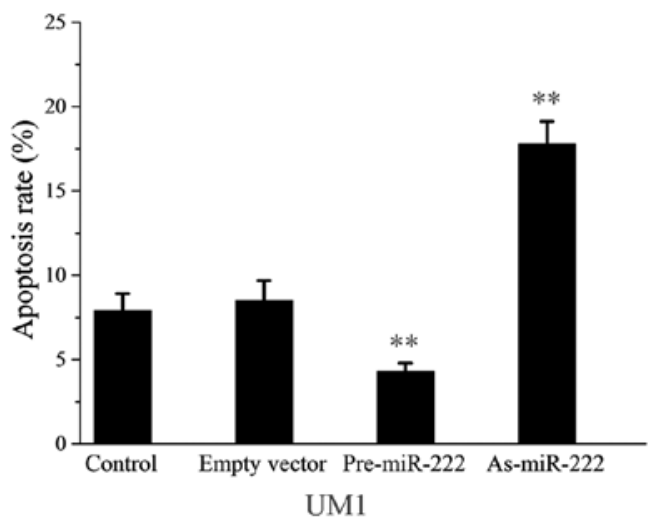

Figure 6. Effect of miR-222 on the apoptosis of OSCC cells. The apoptosis rates of (A) Tca8113 and (B) UM1 cells in the different treatment groups were analyzed by flow cytometry $48 \mathrm{~h}$ after treatment with Pre-miR-2222, As-miR-222 and the empty vector. Annexin V-PI analysis showed that the apoptosis index was highest in the As-miR-2222 group, which was at a significantly higher rate than the other three groups $(\mathrm{P}<0.01)$. Cells transfected with Pre-miR-222 presented a lower apoptosis index than the other three groups $(\mathrm{P}<0.01)$. OSCC, oral squamous cell carcinoma.

proliferation, differentiation, apoptosis and death (15-19) as well as in the process of OSCC and head and neck squamous cell carcinoma (HNSCC) (20-22). As a member of the oncomiRs, miR-222 has a seed sequence, which is a short evolutionarily conserved region through with miRNAs bind its target sites in
mRNA 3'UTRs, indicating an important role in coordinated regulation and function. Some studies have reported that miR-222 may induce cell growth and cell cycle progression by targeting p27 and p57 (3-5,23,24). Moreover, several genes have been found to be common targets of miR-222, such as 
p27, Bmf and PTEN $(4,5,25)$. One study showed that miR-222 may inhibit cell apoptosis in human glioma cells by targeting the pro-apoptotic gene, PUMA $(26,27)$. In the present study, we report the modulating effect of miR-222 on the pro-apoptotic gene PUMA by directly targeting the 3'UTR of PUMA mRNA in OSCC (Tcal8113 and UM1) cell lines. These results may have implications in the pathogenesis of OSCC.

PUMA, which is also named $\mathrm{Bcl}-2$ binding component 3 (BBC3), is a newly discovered tumor suppressor which was induced by the p53 tumor suppressor or other apoptotic stimuli and was found to possess a powerful pro-apoptotic effect. PUMA belongs to the BH3-only subfamily of the Bcl-2 protein family. This gene encodes two $\mathrm{BH} 3$ domain-containing proteins (PUMA- $\alpha$ and PUMA- $\beta$ ), with similar activities, and they bind to $\mathrm{Bcl}-2$ and localize to the mitochondria to induce cytochrome $c$ release to promote apoptosis $(6,28)$. Similar to all the other $\mathrm{BH} 3$-only proteins, PUMA promotes apoptosis through binding to and neutralizing pro-survival members of the Bcl-2 family (28). PUMA induces apoptosis through both p53-dependent and non-p53-dependent pathways and these features make PUMA a particularly potent effector of apoptosis. In the present study, upregulation of the pro-apoptosis protein PUMA and downregulation of the apoptotic protein Bcl-2 were observed following treatment with As-miR-222. Furthermore, bioinformatics and luciferase assays showed that miR-222 may modulate PUMA expression by directly targeting the binding site within the 3'UTR (5). These findings suggest that PUMA is probably directly regulated by miR-222.

The integration of various therapeutic strategies is a trend in current gene therapy to induce synergistic effects $(29,30)$. In the present study, we demonstrated that PUMA gene expression in OSCC cell lines was significantly upregulated at both the mRNA and protein levels by transfection with As-miR-222. We also found that As-miR-222 transfection may obviously upregulate PUMA protein expression and significantly inhibit the growth of Tca8113 and UM1 cells by reducing cell proliferation and promoting cell apoptosis. However, cells transfected with Pre-miR-222 exhibited an opposite trend of expression, and no significant differences between the empty vector transfection group and control group were noted. The results indicate that there was a negative correlation between the expression of PUMA and miR-222, and that the modulation of miR-222 activity may regulate the expression of the PUMA gene, thus affecting the biological behavior of OSCC cells. PUMA is a novel target of miR-222 and may be a critical therapeutic target for OSCC intervention.

\section{Acknowledgements}

The present study was supported by the National Natural Sciences Foundation of China (grant nos. 30973340 and 81272554), the Guangdong Sciences and Technology Project (grant nos. 2011B050400030 and 2012B031800387), and the Guangdong Natural Sciences Foundation (grant nos. 9151008901000187 and S2011020003247).

\section{References}

1. Skaftnesmo KO, Prestegarden L, Micklem DR and Lorens JB MicroRNAs in tumorigenesis. Curr Pharm Biotechnol 8: 320-325, 2007
2. Shi L, Cheng Z, Zhang J, Li R, Zhao P, Fu Z and You Y: Hsa-mir$181 \mathrm{a}$ and hsa-mir-181b function as tumor suppressors in human glioma cells. Brain Res 1236: 185-193, 2008.

3. le Sage C, Nagel R, Egan DA, Schrier M, Mesman E, Mangiola A, Anile C, Maira G, Mercatelli N, Ciafrè SA, Farace MG and Agami R: Regulation of the $\mathrm{p} 27^{\mathrm{Kip} 1}$ tumor suppressor by miR-221 and miR-222 promotes cancer cell proliferation. EMBO J 26: 3699-3708, 2007.

4. Zhang C, Kang C, You Y, Pu P, Yang W, Zhao P, Wang G, Zhang A, Jia Z, Han L and Jiang H: Co-suppression of miR-221/222 cluster suppresses human glioma cell growth by targeting $\mathrm{p} 27^{\mathrm{kip} 1}$ in vitro and in vivo. Int J Oncol 34: 1653-1660, 2009.

5. Zhang C-Z, Han L, Zhang, A-L, Fu, Y-C, Yue, X, Wang G-X Jin Z-F, Pu P-Y, Zhang Q-Y and Kang C-S: MicroRNA-221 and microRNA-222 regulate gastric carcinoma cell proliferation and radioresistance by targeting PTEN. BMC Cancer 10: 367, 2010.

6. Nakano K and Vousden KH: PUMA, a novel proapoptotic gene, is induced by p53. Mol Cell 7: 683-694, 2001.

7. Avila JL, Grundmann O, Burd R and Limesand KH: Radiationinduced salivary gland dysfunction results from p53-dependent apoptosis. Int J Radiat Oncol Biol Phys 73: 523-529, 2009.

8. Labi V and Villunger A: PUMA-mediated tumor suppression: a tale of two stories. Cell Cycle 9: 4269-4275, 2010.

9. Zhang C, Zhang J, Zhang A, Wang Y, Han L, You Y, Pu P and Kang C: PUMA is a novel target of miR-221/222 in human epithelial cancers. Int J Oncol 37: 1621-1626, 2010.

10. Livak KJ and Schmittgen TD: Analysis of relative gene expression data using real-time quantitative PCR and the $2^{-\Delta \Delta C_{\mathrm{T}}}$ method. Methods 25: 402-408, 2001.

11. Tran N, O'Brien CJ, Clark J and Rose B: Potential role of microRNAs in head and neck tumorigenesis. Head Neck 32: 1099-1111, 2010.

12. Chiang WF, Hung PS, Liu SY, Yuan TC, Chang KW, Chen YP, Liu YC and Lin SC: Increase of ZASC1 gene copy number in recurrent oral carcinoma. Oral Dis 17: 53-59, 2011.

13. Childs G, Fazzari M, Kung G, Kawachi N, Brandwein-Gensler M, McLemore M, Chen Q, Burk RD, Smith RV, Prystowsky MB, Belbin TJ and Schlecht NF: Low-level expression of microRNAs let-7d and miR-205 are prognostic markers of head and neck squamous cell carcinoma. Am J Pathol 174: 736-745, 2009.

14. Liu CJ, Tsai MM, Hung PS, Kao SY, Liu TY, Wu KJ, Chiou SH, Lin SC and Chang KW: $m i R-31$ ablates expression of the HIF regulatory factor FIH to activate the HIF pathway in head and neck carcinoma. Cancer Res 70: 1635-1644, 2010.

15. Bartel DP: MicroRNAs: genomics, biogenesis, mechanism and function. Cell 116: 281-297, 2004.

16. Meister G and Tuschl T: Mechanisms of gene silencing by double-stranded RNA. Nature 431: 343-349, 2004.

17. Bagga S, Bracht J, Hunter S, Massirer K, Holtz J, Eachus R and Pasquinelli AE: Regulation by let-7 and lin-4 miRNAs results in target mRNA degradation. Cell 122: 553-563, 2005.

18. Giraldez AJ, Mishima Y, Rihel J, Grocock RJ, Van Dongen S, Inoue K, Enright AJ and Schier AF: Zebrafish miR-430 promotes deadenylation and clearance of maternal mRNAs. Science 312: 75-79, 2006.

19. Wu L, Fan J and Belasco JG: MicroRNAs direct rapid deadenylation of mRNA. Proc Natl Acad Sci USA 103: 4034-4039, 2006.

20. Kozaki K, Imoto I, Mogi S, Omura K and Inazawa J: Exploration of tumor-suppressive microRNAs silenced by DNA hypermethylation in oral cancer. Cancer Res 68: 2094-2105, 2008.

21. Lo WL, Yu CC, Chiou GY, Chen YW, Huang PI, Chien CS, Tseng LM, Chu PY, Lu KH, Chang KW, Kao SY and Chiou SH: MicroRNA-200c attenuates tumour growth and metastasis of presumptive head and neck squamous cell carcinoma stem cells. J Pathol 223: 482-495, 2011.

22. Lajer CB, Garnæs E, Friis-Hansen L, Norrild B, Therkildsen MH, Glud M, Rossing M, Lajer H, Svane D, Skotte L, Specht L, Buchwald C and Nielsen FC: The role of miRNAs in human papilloma virus (HPV)-associated cancers: bridging between HPV-related head and neck cancer and cervical cancer. Br J Cancer 106: 1526-1534, 2012.

23. Fornari F, Gramantieri L, Ferracin M, Veronese A, Sabbioni S, Calin GA, Grazi GL, Giovannini C, Croce CM, Bolondi L and Negrini M: MiR-221 controls CDKN1C/p57 and CDKN1B/p27 expression in human hepatocellular carcinoma. Oncogene 27: 5651-5661, 2008.

24. Medina R, Zaidi SK, Liu CG, Stein JL, van Wijnen AJ, Croce CM and Stein GS: MicroRNAs 221 and 222 bypass quiescence and compromise cell survival. Cancer Res 68: 2773-2780, 2008. 
25. Gramantieri L, Fornari F, Ferracin M, Veronese A, Sabbioni S, Calin GA, Grazi GL, Croce CM, Bolondi L and Negrini M: MicroRNA-221 targets Bmf in hepatocellular carcinoma and correlates with tumor multifocality. Clin Cancer Res 15: 5073-5081, 2009.

26. Zhang C, Han L, Zhang A, Yang W, Zhou X, Pu P, Du Y, Zeng H and Kang C: Global changes of mRNA expression reveals an increased activity of the interferon-induced signal transducer and activator of transcription (STAT) pathway by repression of miR-221/222 in glioblastoma U251 cells. Int J Oncol 36: $1503-1512,2010$.

27. Zhang CZ, Zhang JX, Zhang AL, Shi ZD, Han L, Jia ZF, Yang WD, Wang GX, Jiang T, You YP, Pu PY, Cheng JQ and Kang CS: MiR-221 and miR-222 target PUMA to induce cell survival in glioblastoma. Mol Cancer 9: 229, 2010.
28. Chen L, Willis SN, Wei A, Smith BJ, Fletcher JI, Hinds MG, Colman PM, Day CL, Adams JM and Huang DC: Differential targeting of prosurvival $\mathrm{Bcl}-2$ proteins by their $\mathrm{BH} 3$-only ligands allows complementary apoptotic function. Mol Cell 17: 393-403, 2005.

29. John-Aryankalayil, M, Palayoor, ST, Cerna D, Simone CB II, Falduto MT, Magnuson SR and Coleman CN: Fractionated radiation therapy can induce a molecular profile for therapeutic targeting. Radiat Res 174: 446-458, 2010.

30. Marignol L, Robson, T, McCarthy HO, Worthington J, Murray MM, Hollywood D, Lawler M and Hirst DG: The tissue plasminogen activator gene promoter: a novel tool for radiogenic gene therapy of the prostate? J Gene Med 9: 1032-1038, 2008. 